\title{
What determines drop out in prospective studies of coronary heart disease risk factors between youth and young adulthood: the Young Hearts Study
}

\author{
F J van Lenthe, C A Boreham, J W R Twisk, M J Savage, L Murray, G Davey Smith
}

The growing recognition of the importance of early life factors in the development of coronary heart disease (CHD) may increase prospective research in this period of life. From a methodological point of view, drop out of subjects, particularly when this is selective, is a major threat to the validity of the results. Specific information about determinants of drop out in current studies may therefore yield important information for future studies. We have analysed risk of drop out in the Young Hearts Project, a prospective study in Northern Ireland. ${ }^{1}$

Department of Public

Health, Erasmus

University Rotterdam, PO Box 1738, 3000 DR

Rotterdam, the

Netherlands

F J van Lenthe

Applied Medical

Sciences and Sports

Studies, University of

Ulster, Northern

Ireland

C A Boreham

Institute for Research

in Extramural

Medicine, Vrije

Universiteit

Amsterdam, the

Netherlands

J W R Twisk

Department of Child

Health, Institute of

Clinical Sciences,

Royal Victoria

Hospital, Belfast,

Northern Ireland

M J Savage

Department of

Epidemiology and

Public Health, Queen's

University of Belfast,

Northern Ireland

L Murray

Department of Social Medicine, University of Bristol, United

Kingdom

G Davey Smith

Correspondence to:

Dr van Lenthe

(vanlenthe@mgz.fgg.eur.nl)

Accepted for publication 16 March 2001

\section{Methods and Results}

The Young Hearts Project is a prospective cohort study, which started in two cohorts of 12 year old boys $(n=251)$ and girls $(n=258)$ and 15 year old boys $(n=252)$ and girls $(\mathrm{n}=254)$ in Northern Ireland (overall response rate $78 \%$ ) in 1988 . Its aim and design have been described elsewhere in detail. ${ }^{1}$ In 1992/93 these children were re-examined under identical conditions. In 1997 the third wave of data collection was carried out in participants mean age 21 years (cohort 1 ) and 24 years (cohort 2 ). In cohort 1 and 2 data were obtained from $49.9 \%$ (boys: $n=135$; girls: $n=119$ ) and $46.4 \%$ (boys: $n=116$; girls: $n=119$ ) respectively of the subjects included at baseline. We created a dichotomous variable thereby distinguishing those at baseline who were still in the study collection in boys and girls 12 and 15 years of age at baseline

$\star$ Values based on tertiles, with missing values included as a separate group. nine years later from those who were in the study initially but who dropped out of the study. Logistic regression analysis was carried out to investigate if less then good perceived general health, sociodemographic (sex and socioeconomic position), biological (birth weight, body height, body weight, sum of four skinfolds, systolic and diastolic blood pressure, total serum cholesterol, high density lipoproteins and cardiorespiratory fitness (measured by the 20 metre shuttle run test) and behavioural (physical inactivity, total energy intake, and intake of fat, cholesterol, fruit and vegetable) risk indicators of coronary heart disease at baseline were associated with risk of drop out. Indicators univariately associated with risk of drop out $(p<0.20)$ were entered in a multivariate analyses; final models were derived using a backward elimination procedure.

Table 1 presents the univariate and multivariate associations for the final models. Consistent findings in both cohorts are the significantly increased odds ratio for those in the manual compared with the non-manual groups. Odds ratios of drop out increased by increasing sum of skinfolds (in cohort 1) and increasing systolic blood pressure (in cohort 2). In both cohorts risk of drop out was associated with birth weight; however, an increased odds ratio was found in subjects in the lower tertile of birth weight in cohort 1 and the upper tertile

Table 1 Determinants of drop out in the Young Hearts Project between the first (1988) and third (1997) wave of data

\begin{tabular}{|c|c|c|c|c|c|}
\hline & \multirow[b]{2}{*}{ Number } & \multicolumn{2}{|l|}{ Univariate } & \multicolumn{2}{|l|}{ Multivariate } \\
\hline & & Odds ratio $(95 \% \mathrm{CI})$ & $p$ & Odds ratio $(95 \% \mathrm{CI})$ & $p$ \\
\hline \multicolumn{6}{|l|}{ Cohort 1 (12 years at baseline) } \\
\hline Socioeconomic position & & & 0.00 & & 0.00 \\
\hline Non-manual & 280 & 1.00 & & 1.00 & \\
\hline Manual & 178 & $1.97(1.35,2.88)$ & & $1.93(1.31,2.84)$ & \\
\hline Missing & 51 & $3.79(1.96,7.32)$ & & $3.78(1.93,7.37)$ & \\
\hline Birth weight $(100 \mathrm{~g})$ & & & 0.01 & & 0.03 \\
\hline Normal & 156 & 1.00 & & 1.00 & \\
\hline Low $(<3118 \mathrm{~g})^{\star}$ & 155 & $1.22(0.78,1.90)$ & & $1.12(0.70,1.77)$ & \\
\hline High $(>3540 \mathrm{~g})$ & 154 & $0.62(0.40,0.98)$ & & $0.62(0.39,0.99)$ & \\
\hline Missing & 44 & $1.51(0.76,2.99)$ & & $1.45(0.72,2.91)$ & \\
\hline Sum of skinfolds (cm) & 509 & $1.12(1.02,1.23)$ & 0.02 & $1.13(1.02,1.25)$ & 0.02 \\
\hline \multicolumn{6}{|l|}{ Cohort 2 ( 15 years at baseline) } \\
\hline Socioeconomic position & & & 0.01 & & 0.02 \\
\hline Non-manual & 253 & 1.00 & & 1.00 & \\
\hline Manual & 189 & $1.79(1.22,2.62)$ & & $1.75(1.18,2.60)$ & \\
\hline Missing & 64 & $1.45(0.83,2.51)$ & & $1.28(0.72,2.26)$ & \\
\hline Birth weight (100 g) & & & 0.06 & & 0.01 \\
\hline Normal & 155 & 1.00 & & 1.00 & \\
\hline Low $(<3180 \mathrm{~g})^{\star}$ & 157 & $1.33(0.85,2.07)$ & & $1.21(0.76,1.92)$ & \\
\hline High $(>3629 \mathrm{~g})$ & 154 & $1.80(1.15,2.83)$ & & $2.12(1.32,3.41)$ & \\
\hline Missing & 40 & $1.77(0.88,3.60)$ & & $2.18(1.05,4.52)$ & \\
\hline Body height $(\mathrm{cm})$ & 506 & $0.98(0.96,1.00)$ & 0.11 & $0.97(0.94,0.99)$ & 0.05 \\
\hline Systolic blood pressure $(10 \mathrm{~mm} \mathrm{Hg})$ & 506 & $1.29(1.11,1.49)$ & 0.00 & $1.36(1.16,1.59)$ & 0.00 \\
\hline
\end{tabular}


in cohort 2. There seemed to no statistically significantly associations between behavioural risk indicators of coronary heart disease and risk of drop out.

\section{Comments}

We described the profile of children at increased risk of drop out. Clearly, there is no information as to whether these children still have the same risk profile in young adulthood. Tracking studies however predict a relative stability in risk factors in age and sex specific populations over time. ${ }^{2}$ Hence, there are reasons to expect that those who dropped out of the study are likely to show the same risk profile in young adulthood as they did in childhood.

In non-response research refusal, illness and having moved to another school were given as main reasons for drop out between the first and second period of measurement. ${ }^{3}$ Unfortunately, no such information is available between the second and third wave of data collection.

In our study, socioeconomic position seemed to be a main determinant of drop out. Interestingly, there is increasing awareness of the need to study socioeconomic inequalities in coronary heart disease from a life course perspective. ${ }^{4}$ Our findings suggest that socioeconomic inequalities in coronary heart disease risk indicators in early life may be underestimated as a consequence of selective drop out by socioeconomic position (and some coronary heart disease risk indicators). While further investigating the life course perspective, researchers need to pay particular attention to those in lower socioeconomic groups with respect to adherence to the study.

Funding: this study is financially supported by the Northern Ireland Chest, Heart and Stroke Association, the British Heart Foundation and Wellcome Trust.

Conflicts of interest: none.

1 Boreham C, Savage JM, Primrose D, et al. Coronary risk factors in schoolchildren. Arch Dis Child 1993;68:182-6.

2 Twisk JWR, Kemper HCG, Mechelen W van, et al. Tracking of risk factors for coronary heart disease over a 14-year period: a comparison between lifestyle and biologic risk factors with data from the Amsterdam Growth and Health Study. Am ₹ Epidemiol 1997;145:888-98.

3 Twisk JWR, Boreham C, Cran G, et al. Clustering of Twisk JWR, Boreham C, Cran G, et al. Clustering of

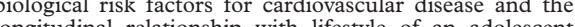
population: The Northern Ireland Young Hearts Project. $\mathcal{F}$ population: The Northern Irelas

4 Davey Smith G, Hart C, Gilles C, et al. Lifetime socioeconomic position and mortality: prospective observational study. BMF 1997;314:547-52

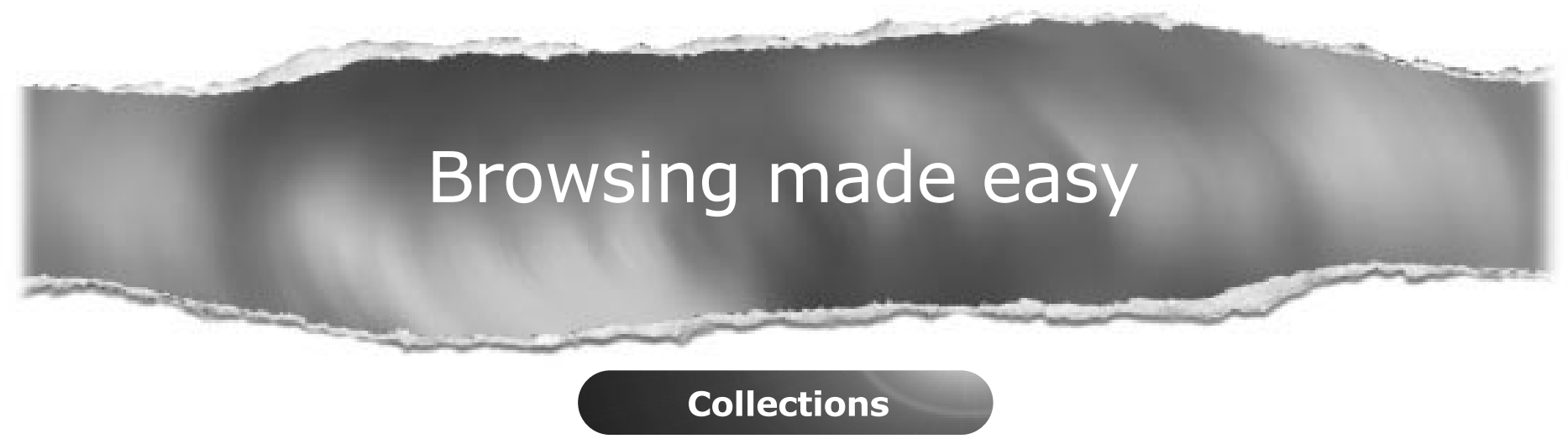

With a single click Collections allows you to find all articles that have been published in your chosen subject. Select from over 200 clinical and non-clinical topic collections and/or cross search other specialist journals, the BMJ and Cochrane Reviews

\section{www.jech.com}

